\title{
POLAR-2: The First Large Scale Gamma-Ray Polarimeter
}

\author{
Merlin Kole* on behalf of the POLAR-2 collaboration ${ }^{\dagger}$ \\ https: //www.astro.unige.ch/polar-2/ \\ University of Geneva \\ E-mail: merlin.kole@unige.ch
}

\begin{abstract}
The mechanisms responsible for the prompt emission of Gamma-Ray Bursts (GRBs) remain poorly understood despite a wealth of measurements performed over the last 5 decades. After detailed measurements of the photon direction, time and energy, recent years have seen the first measurements of the polarization of the prompt emission by two dedicated polarimeters, POLAR and GAP. The typical low levels of polarization observed by POLAR and the temporal evolution of the polarization angle observed by both instruments indicates that time resolved studies are required to constrain theoretical emission models. Additionally a larger sample of GRB polarization measurements is required to answer the remaining open questions on gamma-ray emission in GRBs. The POLAR-2 instrument aims to provide such measurements by having an effective area of one order of magnitude larger than that of POLAR. The detector, proposed by an international collaboration, was recently selected to be launched in 2024. It will then be placed on the China Space Station and operate for at least 2 years. By improving the design of POLAR and scaling up the instrument, an increase in effective area of one order of magnitude compared to POLAR can be achieved. Using the improved design, which includes the replacement of photomultiplier tubes with silicon photomultipliers, as well as other technological upgrades, the instrument is foreseen to perform detailed polarization measurements of 50 GRBs per year. The instrument design will be discussed in detail together with the scientific potential expected from the mission.
\end{abstract}

36th International Cosmic Ray Conference -ICRC2019-

July 24th - August 1st, 2019

Madison, WI, U.S.A.

\footnotetext{
${ }^{*}$ Speaker.

${ }^{\dagger}$ for collaboration list see PoS(ICRC2019)1177
} 


\section{GRBs}

Gamma-Ray Bursts (GRBs) are detected as bright bursts of $\mathrm{X} / \gamma$-rays which lasts from hundreds of milliseconds up to hours. This emission, called the prompt-emission, is followed by longer lasting afterglow emitted at lower energies such as in the optical and radio bands. GRBs can be divided into two different types, short bursts which last below 2 seconds and long burst which last longer than 2 seconds. The short burst were recently shown to originate from the merger of two neutron stars thanks to the measurement of a gravitational counterpart to GRB 170817A by the LIGO and VIRGO collaboration [1]. For the long bursts strong evidence exists for their origin to be connected to the collapse of massive stars [2]. The high energy emission from both types of GRBs are thought to originate from two relativistic jets, causing GRBs to only be observed if the jet is pointed towards the observer, however, with the discovery of 170817A it appears that also off-axis GRBs can potential be observed.

While the flux, spectral and timing measurements of $\gamma$-ray emission from astrophysical sources has been studied extensively, few measurements of the polarization degree and angle of these photons exist, despite the wealth of information held by these two parameters. In particular, for GRBs the polarization properties of the $\gamma$-ray emission are largely dependent on the involved emission processes, magnetic fields and structure of the emitting jets [3]. Theoretical models predicting the majority of the prompt emission to be the result of synchrotron radiation, indicating magnetic fields in the jets, predict polarization degrees as high as 50\% [4]. If the magnetic fields are highly ordered the average polarization degree will be $40 \%$ within a sample of GRBs. In other models the prompt emission is a result of inverse-Compton scattering. Such models predict the polarization degree to be high in the case of a large angle between the observer and the jet while typical GRBs will be unpolarized. A strong negative correlation is therefore predicted between the observing angle and polarization degree [5].

The polarization angle, which one automatically observes when measuring the polarization degree, can be used to probe the internal structure of the jet. While some models predict an axisymmetric structure others predict a jet consisting of several mini-jets. Axi-symmetric models makes the strong prediction that the polarization angle can only change during the observation by $90^{\circ}$, or it does not change at all [6]. Any random changes in the polarization angle would disfavor both such axi-symmetric models which include synchrotron models with ordered magnetic fields as well as photospheric emission models. Measurements of the polarization angle and its evolution are therefore powerful probes for GRB jets.

\section{Gamma-Ray Burst Polarimetry Measurements}

Due to the large scientific potential of the polarization parameters a number of instruments dedicated to performing polarimetry on astrophysical sources have been proposed in recent years. Currently several new dedicated polarimeters in the X/ $\gamma$-ray energy range are either under construction or in the proposal phase, some examples are X-Calibur [7], LEAP [8], eXTP [9] and IXPE [10]. This decade has also seen a range of successful polarization measurements of different astrophysical sources by instruments such as GAP [11][12], COSI [13], PoGOLite [14] and most recently by POLAR [15], [16]. Although GRB polarization measurements by the AstroSAT collab- 
oration have been reported, the analysis results have thus far not been published in peer-reviewed journals. These preliminary results do, however, indicate that a set of polarization measurements from this instrument can be expected in the near future.

The most extensive set of GRB polarization measurements thus far comes from the POLAR instrument which is described in detail in [17]. POLAR was a dedicated polarimeter launched as part of the Chinese Tiangong 2 (TG-2) Space Lab in September 2016. The instrument made use of a segmented scintillator array of $40 \times 40$ plastic bars, optimized for performing polarization measurements in the $50-500 \mathrm{keV}$ energy range. The plastic scintillators were readout in groups of 64 using Multi-Anode PMTs (MAPMTs) each of which had its own front-end electronics (FEE). The combination of the FEE, the MAPMT and the 64 bars together with the mechanics is referred to as a detector module. While operational between September 2016 and April 2017, when the instrument suffered from a problem with the High Voltage Power Supply (HVPS) at least 55 GRBs were detected. The polarization results of 5 GRBs, selected based on their brightness, incoming angle and detection by other instruments, were recently published [15]. The polarization for all 5 GRBs was found to be low and compatible with an unpolarized flux while upper limits ranging from polarization degrees of $28 \%$ to $67 \%$ were reported. Although these measurements appear to disfavor synchrotron models with highly ordered magnetic fields, time resolved studies of the brightest GRB in the catalog found an evolution of the polarization angle. Using time resolved analysis it was found that this single pulse GRB, 170114A, was polarized by approximately $30 \%$ with a polarization angle changing by approximately $60^{\circ}$ degrees between the 3 time bins studied. An unpolarized origin was excluded with $99.7 \%$ confidence. The time evolution of the polarization angle could be the cause of the low polarization degrees measured for all 5 GRBs as detailed time resolved analyzed of the other 4 GRBs was not possible due to a lack of statistics. A further in depth analysis of GRB 170114A where POLAR data was combined with that spectral data from Fermi-GBM confirmed the time evolution of the polarization angle. Using a total of 9 time bins a continuous evolution was observed, see figure 1 while the spectrum was found to be compatible with that of synchrotron emission [16]. Although both the spectrum and the polarization degree appear to favor synchrotron emission a satisfying theoretical explanation for the evolution of the polarization angle is still missing.

While another 50 GRBs measured by POLAR are currently being analyzed by the collaboration these first results indicate the need for higher precision measurements in the future. Firstly, in order to produce large catalogs of polarization measurements with ample precision more precise instrumentation is required. Such an instrument could furthermore produce precision measurements of specific GRBs allowing to perform both detailed time resolved analysis as well as for energy resolved analysis. Based on this insight the POLAR-2 instrument was selected to be launched towards the China Space Station in 2024 by CSU and UNOOSA in June 2019.

\section{Instrument Design}

\subsection{Overall Requirements}

The design goal of the POLAR-2 instrument is to gain one order of magnitude in effective area compared to the POLAR detector. This is achieved firstly both by scaling up the instrument 

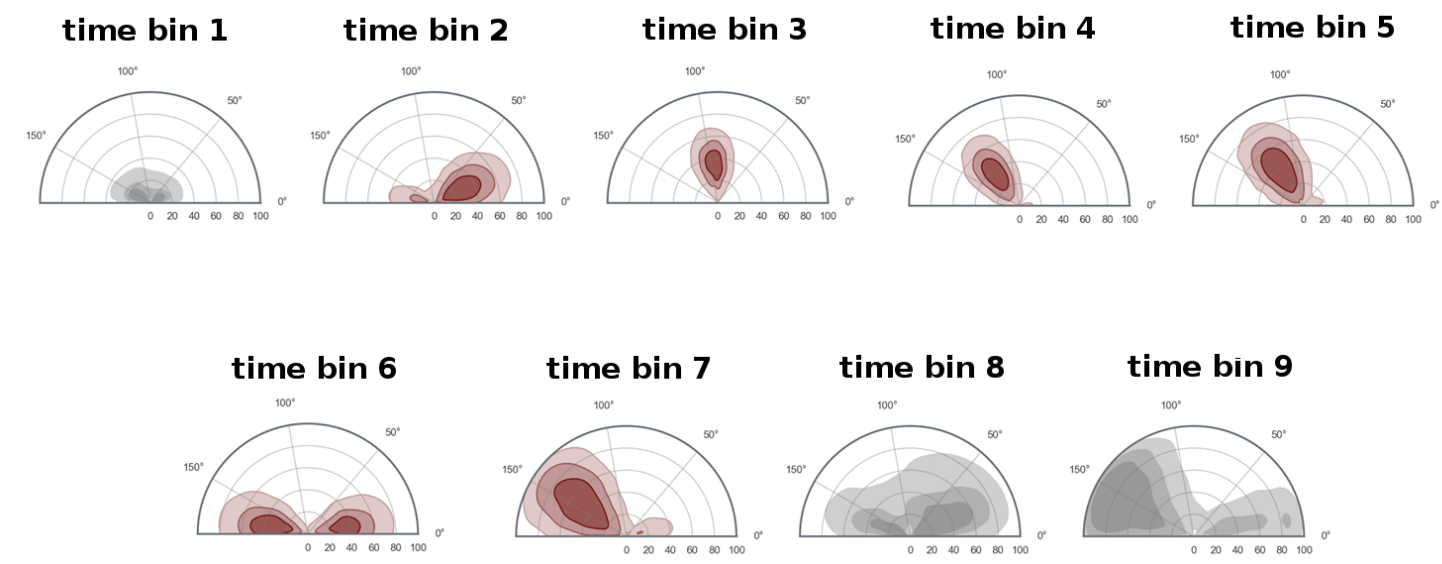

Figure 1: The polarization measurements of 9 different time bins of GRB 170114A using a combination of the POLAR and Fermi-GBM data as presented in [16]. The polarization degree is plotted along the radial axis whereas the polarization angle is along the theta axis. The polarization angle can be seen to vary significantly during the GRB while the polarization degree remains around $40 \%$ throughout the GRB.

by a factor 4 . Additionally several components will be replaced and upgraded in order to improve the scientific performance as well as to mitigate all problems experienced during the operation of POLAR and during its development. For more information on the issues encountered during development the reader is referred to [17]. The overall design remains largely based on that of POLAR which is described in detail in [17]. The POLAR-2 instrument will have a total mass of approximately $100 \mathrm{~kg}$, power consumption of approximately 200 Watts, physical dimensions of $700 \times 700 \times 830 \mathrm{~mm}^{3}$ and produce $30 \mathrm{GBs}$ of data per day.

\subsection{Polarimeter Design}

Similar to the POLAR design the instrument will have a modular structure. POLAR-2 will consist of a total of 100 detector modules. Photons are detected through their interaction within a plastic scintillator array consisting of bars with a cross section of $6 \times 6 \mathrm{~mm}$ with a baseline design length of $178 \mathrm{~mm}$. The polarization of the photons is inferred by measuring their azimuthal Compton scattering angle. For a polarized flux, the distribution of the Compton scattering angles, referred to as a modulation curve, will be modulated with a period of 180 degrees, whereas for an unpolarized flux it will be uniform. Such a measurement requires the photon to initially undergo a Compton scattering interaction in one of the scintillators followed by a secondary interaction in another scintillator. The azimuthal Compton scattering angle is then defined by the location of the two scintillators. A typical event which can be used for polarization analysis therefore consists of at least two interactions within the coincidence window, which is foreseen to be $100 \mathrm{~ns}$ as in POLAR. Events consisting of a single bar triggering can therefore be discarded as can events with high multiplicities as these are typically the result of cosmic rays.

Contrary to POLAR where the scintillators were read-out using a multi-anode PMT (MAPMT), the plastic scintillators in POLAR-2 are read out using a silicon photomultiplier array of 8 by 8 channels. This change serves two main purposes. Firstly, it allows to remove the need for a high voltage power supply which are both difficult to develop for space applications and the only com- 
ponent on the POLAR mission which failed during the 2.5 year flight. Secondly the SiPM readout allows to lower the low energy detection threshold as the number of photo-electrons per deposited $\mathrm{keV}$ in the scintillators can be increased to 1.5 from 0.75 photoelectrons $/ \mathrm{keV}$ in the case of POLAR. Therefore the effective area, especially in the low energy region, increases significantly. The typical downside of SiPM use over PMTs is the dark noise. This however, is greatly reduced in POLAR-2 by operating the instrument at $-10^{\circ} \mathrm{C}$ through passive cooling. Based on preliminary calculations it is foreseen to operate the instrument with a threshold of 4 photoelectrons for both bars inside of a multiplicity 2 event. This ensures a low detection threshold of around $2 \mathrm{keV}$, while keeping the chance coincidence rate from dark noise within a single module in the order of 10's of $\mathrm{Hz}$ at room temperature. For single multiplicity events, which can be used for spectral studies as well as for pulsar observations, the threshold is increased to approximately 7 photo-electrons. At lower temperatures these thresholds can be reduced.

Each module contains its own front-end electronics (FEE) used to read-out the SiPM array, to perform trigger decisions on the modular level and to communicate with the back-end electronics. The baseline design of the FEE contains 2 CITIROC ASIC chips [18], used for reading out the $\mathrm{SiPM}$ array, and a relatively simply FPGA used to handle the trigger logic and communication. The pitch distance between channels is equal to that of the MAPMTs used in POLAR, allowing to keep much of the mechanical design equal on the modular level. A schematic image of a full module is shown in figure 2. In this image the SiPM array can be seen together with the scintillator bars, the carbon fiber cover, a plastic grid which holds the scintillators in place and the front-end electronics coupled directly to the SiPM. A rubber damper is placed between the scintillators and the top of the carbon fiber cover. The damping materials can be significantly reduced compared to those in POLAR allowing for a reduction in the amount of dead material above the scintillators. This is possible as POLAR-2 will have lower requirements for shock and vibration resistance compared to the POLAR instrument (which was designed to sustain shocks up to $800 \mathrm{G}$ ), and as the SiPM array is more robust compared to a MAPMT.

The arrangement of the detector modules is indicated in figure 2 where a top carbon fiber cover, which serves to stop low energy charged particles, has been made transparent. The arrangement of the modules, which unlike POLAR is not fully square, is mainly based on the installation requirements for the CSS. Similarly the large blue volume below the polarimeter is a mainly empty box allowing for POLAR-2 to be installed on the CSS using a robotic arm which connects to the red piece on the side of POLAR-2. The large empty volume will however also serve to radiate away heat thereby providing an operating temperature of $-10^{\circ} \mathrm{C}$.

Apart from the polarimeter which forms the main instrument on POLAR-2 the payload will contain a total of 12 spectrometers. The design of the spectrometers will be based on those used in GECAM [19]. The goal of these spectrometers, which are oriented such that their field of view covers half the sky, is to ensure detailed spectral and location measurements exist for all GRBs observed by POLAR-2. The POLAR mission detected several GRBs which were not observed by $\gamma$-ray spectrometers or for which a location measurement within 1 degree does not exists. As the lack of such measurements induces significant systematic errors on the polarization measurement it was decided that POLAR-2 should not rely on other $\gamma$-ray detectors operating at the time for such information. Optimization studies of the spectrometer design are currently ongoing. 

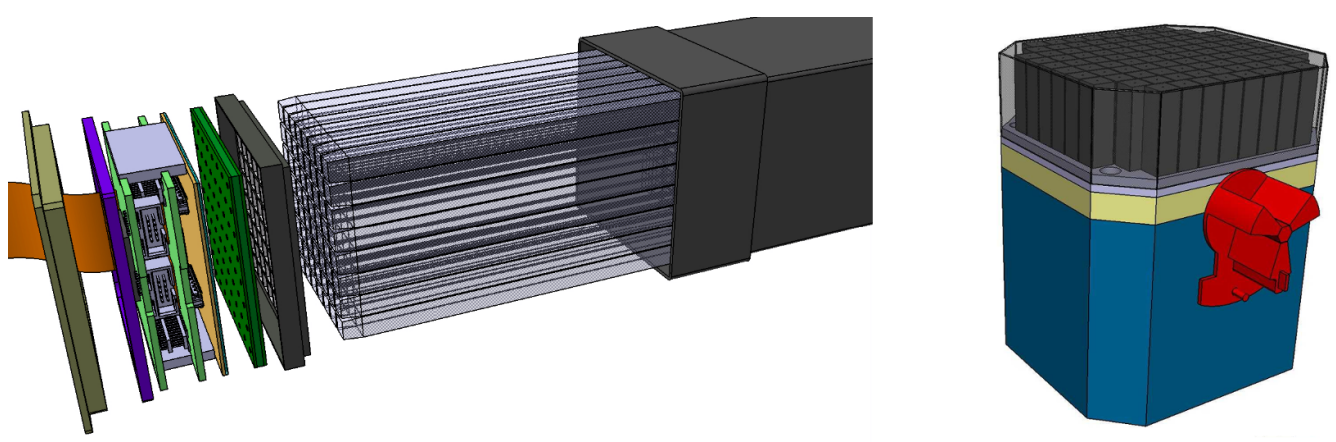

Figure 2: Left: Schematic representation of a module in POLAR-2. The carbon cover is shown in black, here covering the top parts of the transparent plastic scintillators. Below the black grid used to hold the scintillators in place is shown, below this the SiPM array is shown in green above the FEE electronics containing also the power supply for the SiPM and connectors for communication with the back-end electronics and power. Right: The schematic representation of the full POLAR-2 instrument. The 100 modules can be seen in black underneath the semi-transparent carbon cover. The red object as well as the blue box at the bottom are mechanical parts required for mounting on the CSS.

\subsection{Simulation Based Scientific Performance of POLAR-2}

Preliminary studies of the POLAR-2 performance were performed using a MC package based on the POLAR MC software as presented in [20]. The effective area and the $M_{100}$ (a parameter used to express the sensitivity to polarization) as a function of energy were simulated for a typical GRB using the current baseline design of POLAR-2. The incoming angle of the GRB here was taken to be $26^{\circ}$ off-axis, equal to that of GRB 170114A observed by POLAR. The simulations were performed for 3 different instrument configurations, the original POLAR instrument, the POLAR instrument increased in size by a factor of 4 (referred to as POLARx4) and finally POLAR-2. In the POLAR-2 simulations the POLAR design for detector modules is used while the PMTs are replaced by SiPMs. The performance of the SiPMs is based on that of the data sheets of the S13361-6075 MPPC from Hamamatsu as well as on preliminary lab studies. The replacement of the PMTs by SiPMs was taken into account in the simulation software by increasing the photon detection efficiency (PDE) from 0.2 to 0.5 , decreasing the MAPMT/SiPM resolution from 0.6 to 0.1 , reducing the optical cross talk by one order of magnitude, removing the common noise and setting the threshold to approximately 6 photo-electrons, which is a pessimistic assumption. As studies on the optimization of the scintillator bars design and the mechanical design of the modules, where a reduction in the amount of dead material is foreseen, are still ongoing, the same designs as in POLAR were used here. Further improvements in the performance can therefore be expected.

The results of the effective area study are presented in figures 3 . The ratio between the effective area of POLAR and POLAR-2 is shown in the right panel of the figure. The results show that even using relatively pessimistic assumptions and analysis techniques optimized for POLAR the effective area is significantly larger especially in the low energy region (below $100 \mathrm{keV}$ ) as a result of the SiPM usage. At higher energies, an improvement can also be seen compared to POLARx4 which is a result of the larger dynamic range of the FEE. In the POLAR FEE the upper limit of the dynamic range is limited to approximately $300 \mathrm{keV}$, for POLAR-2 this can be increased to approximately $1 \mathrm{Mev}$. For the modulation factor no significant changes with respect to those 
of POLAR were observed. This is likely a result of the use of event selection techniques which were optimized for the POLAR detector. Optimizing the event selection as well as reducing the amount of dead material in the detector in the future are expected to result in improvements in the modulation factor as well.

One of the 5 most precise measurement performed by POLAR was for a moderately bright GRB: 170206A. This GRB lasted 2 seconds and had a fluence of approximately $1.3 \times 10^{-5} \mathrm{erg} / \mathrm{cm}^{2}$ in the $10-1000 \mathrm{keV}$ band [21]. Here we use 170206A to study the improvement in performance for POLAR-2 compared to POLAR using both effective area studies and preliminary background studies for POLAR-2. For these studies the spectrum and fluence of GRB 170206A was used together with the instrument responses of POLAR and POLAR-2 to simulate a measurement. For these simulations a real background sample from the POLAR data was used to simulate the background, for POLAR-2 a background rate of a factor 3.5 higher than that of POLAR was used. Figure 4 shows the likelihood distributions resulting from these simulations for when the GRB is unpolarized for POLAR and POLAR-2 (top) and for the scenario where the GRB is $10 \%$ polarized (bottom). It can be clearly observed that with POLAR it was not possible to distinguish between an unpolarized and a $10 \%$ polarized GRB with this fluence. POLAR-2 can however clearly distinguish these two scenarios with more than $99 \%$ confidence. It was additionally shown that POLAR-2 will be capable of performing measurements with an equal precision as that published by POLAR for GRB $170206 \mathrm{~A}$ for GRBs with a fluence of $2 \times 10^{-6} \mathrm{erg} / \mathrm{cm}^{2}$. POLAR-2 is therefore expected to produce a total of 50 measurements per year with a precision higher or equal to the 5 GRB polarization measurements published by POLAR to date.
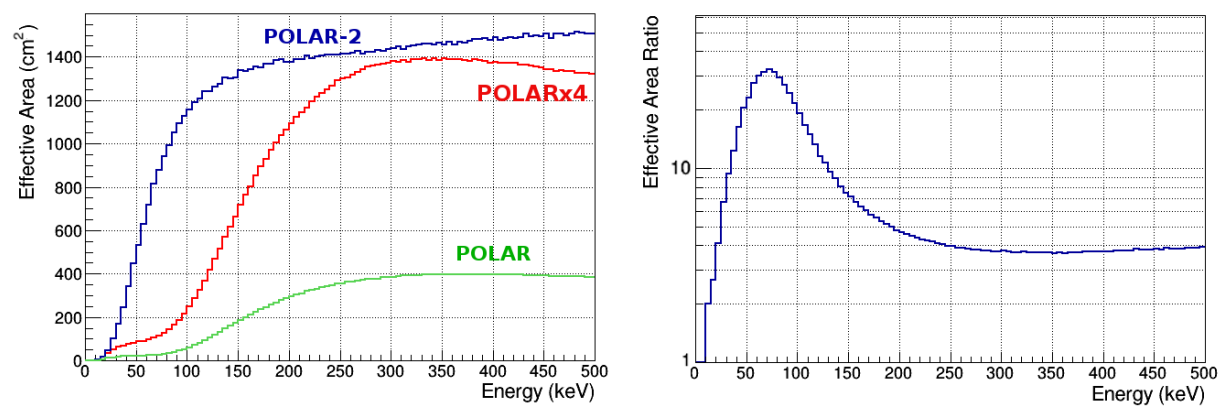

Figure 3: The effective area (for polarization events) as a function of incoming photon energy of POLAR-2 (blue) compared to that of POLAR (green) and that of a version of POLAR with a 4 times larger area (red) on the left. The ratio of the effective area of POLAR-2 over that of POLAR as a function of energy can be seen on the right. It can be seen that especially at low energies a significant improvement is found.

\section{References}

[1] B.P. Abbott et al., ApJL 848 (2019)

[2] S.E. Woosley Astrophys. J. 405 (1993) 273-277

[3] Toma, K. et al. Astrophys. J. 698 (2009) 1042-1054

[4] M. Luytikov et al., ApJ. 597 (2003) 998

[5] S. Dado et al., Proc. Vulcano Conf. arXiv:astro-ph/0701294v2 


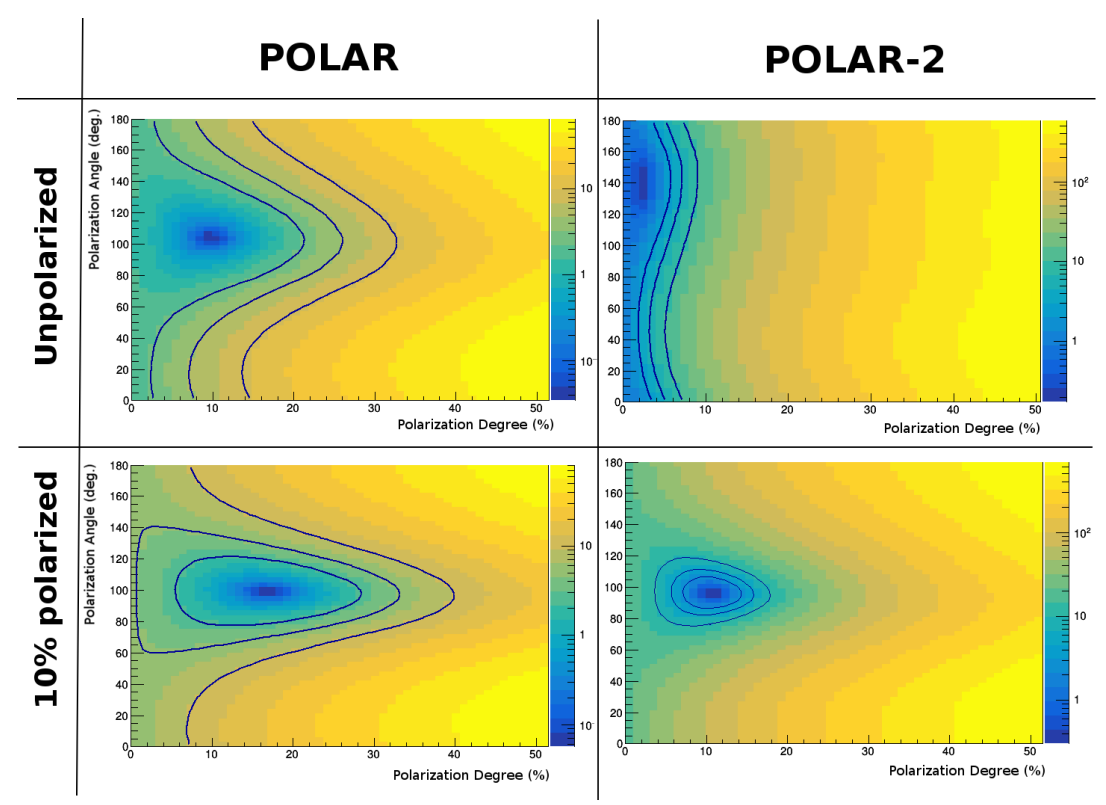

Figure 4: The simulated likelihood distributions acquired for an unpolarized GRB with a fluence of $1.3 \times$ $10^{-5} \mathrm{erg} / \mathrm{cm}^{2}$ for both POLAR (top left) and POLAR-2 (top right) and that for a $10 \%$ polarized GRB with equal fluence in the bottom. The 1 and $2 \sigma$ confidence intervals are shown with blue lines as well as the 99\% confidence line. It can be seen that the simulated distributions for POLAR both match the measured results for GRB 170206A [15]. With POLAR for GRB 170206A it was not possible to determine whether the GRB was polarized by $10 \%$ or unpolarized. With POLAR-2 such a discrimination is possible with more than $99 \%$ confidence.

[6] C. Lundman et al., MNRAS 428 (2013) 2430

[7] Q. Guo et al., ApJ. 41 (2013) 63

[8] M.L. McConnell et al., Huntsville GRB Workshop Proc. 1962 (2016) 4051

[9] S.N. Zhang et al., SPIE proc. Vol 9905 arXiv:1607.08823

[10] M.C. Weisskopf et al., Res. in Phys. 6 (2016) 1179-1180

[11] D. Yonetoku et al., ApJ. 743 (2011) 30

[12] D. Yonetoku et al., ApJ. 758 (2012) 1

[13] C.A. Kierans et al., Proc. INTEGRAL Conf. arXiV:1701.05558v1

[14] M. Chauvin et al., MNRAS 456 (2016) 84-88

[15] S.N. Zhang, M. Kole et al., Nat. Astro. 3 (2019) 258â̆

[16] J.M. Burgess et al., A\&A 627 (2019), A105, arXiv:1901.04719

[17] N. Produit et al., Nucl. Instr. and Meth. A 550 (2005) 616.

[18] https://www.weeroc.com/en/products/citiroc-1a

[19] P. Lv et al. Journ. of Instr. 13 (2018)

[20] M. Kole et al. Nucl. Instrum. Meth. Phys. Res. A. 872 (2017) 28-40

[21] GCN for GRB 170206A. https://gcn.gsfc.nasa.gov/other/170206A.gcn3, accessed 25-June-2019. 